\title{
Propiedades psicométricas del Children Psychological Well-being Scale en población colombiana ${ }^{1}$
}

\section{Psicometric properties of the Children Psychological Well-being Scale in Colombian population}

\author{
Stefano Vinaccia-Alpi ${ }^{2}$, Nazanin Abed ${ }^{3}$, Andrea Ortega-Bechara ${ }^{2}$, \\ Rosangela Raleigh ${ }^{2}$, César Andrés Gómez-Acosta ${ }^{4}$, \\ María Alejandra Vieira², Yuli Jiménez², \\ Lina López-Pico y Brayan Vinasco ${ }^{2}$
}

Citación: Vinaccia-Alpi, S., Abed, N., Ortega-Bechara, A., Raleigh, R., Gómez-Acosta, C.A., Vieira, M.A., Jiménez, Y., López-Pico, L y Vinasco, B. (2020). Propiedades psicométricas del Children Psychological Well-being Scale en población colombiana. Psicología y Salud, 30(2), 265-273. https://doi.org/10.25009/pys.v30i2.2660.

\section{RESUMEN}

\begin{abstract}
Si bien se han desarrollado y validado algunos instrumentos para medir la calidad de vida en adolescentes y adultos colombianos, y en otros países también se han realizado investigaciones en niños; cabe mencionar que, no hay evidencia de estudios similares en Colombia. Por tal motivo, el objetivo de la siguiente investigación fue describir las propiedades psicométricas iniciales de la versión en español del Children Well-Being Scale [CPWS] en una muestra poblacional de 229 niños preescolares colombianos entre 3 y 6 años de edad. Se desarollaron análisis descriptivos, análisis de dimensionalidad de los modelos a través de análisis factorial confirmatorio, análisis de correlaciones de Pearson, y una serie de análisis de varianza y de pruebas $t$ de los factores de la CPWS según la información sociodemográfica de los participantes. Los resultados indicaron que los análisis factoriales confirmatorios realizados apoyan la estructura factorial del CPWs propuesta en la validación original en Irán. Adicionalmente, los indicadores psicométricos obtenidos en la traducción colombiana corresponden con los criterios de bienestar psicológico en niños definidos por la teoría que sustenta este tipo de instrumentos. Finalmente, el CPWS como un cuestionario original, mantiene tres factores: autoconcepto, satisfacción con la vida y resiliencia, con validez y confiabilidad aceptables.
\end{abstract}

Palabras clave: Escala de bienestar psicológicos en niños; Bienestar psicológico; Propiedades psicométricas; Niñez.

\begin{abstract}
Although some instruments have been developed and validated to measure quality of life in Colombian adolescents and adults, and there is a history of measuring this variable with children in the world, there is no evidence from similar studies in Colombia. The objective of the present study was to develop construct validity and reliability of the Spanish version of the Children Well-Being Scale [CPWS] in a population sample of 229 Colombian preschool children ages 3 to 6 years. Through a cross-sectional descriptive and instrumental study, the CPWS questionnaire was applied to parents of preschool children of both sexes of the department of Cordoba, Colombia. Descriptive, dimensionality analyses of the models included confirmatory factor analysis, Pearson
\end{abstract}

\footnotetext{
${ }^{1}$ Los autores agradecen el apoyo de las instituciones universitarias mencionadas que hicieron posible el presente trabajo colaborativo, financiado por la Universidad del Sinú "Elías Bechara Zainum”. Artículo recibido el 8 de junio de 2019 y aceptado el 16 de enero de 2020.

${ }^{2}$ Programa de Psicología, Universidad del Sinú, Carrera 1w 38-153, Montería, Colombia, correos electrónicos: vinalpi47@hotmail.com y stefanovinacci@unisinu.edu.co.

${ }^{3}$ Shadid Beheshti University, Tehran Province, District 1, Daneshjou Boulevard, 19839 69411, Irán.

${ }^{4}$ Fundación Universitaria Sanitas, Ak 68 Núm. 22A-30, Bogotá, Colombia.
} 
correlation analysis, and a series of variance analysis (ANOVA) and t-tests for CPWS factors according to the socio-demographic information of participants. The results first indicated the confirmatory factor analyses performed support the factor structure of the CPWS proposed in the original validation in Iran. In addition, the psychometric indicators obtained in the Colombian translation correspond to the criteria of psychological well-being in children defined by the theory behind this type of instrument. Finally, the CPWS questionnaire, as did the original questionnaire, maintained three factors: self-concept, life satisfaction and resilience, with acceptable validity and reliability.

Key words: Children Well-Being Scale; Psychological wellbeing; Psychometric properties; Childhood.

$\mathrm{E}$ 1 bienestar subjetivo (SWB) es un constructo teórico desarrollado en los años 80 por el psicólogo norteamericano Ed Diener, quien lo define como el proceso que llevan a cabo las personas para alcanzar a experimentar bienestar (Diener, 1984).

En la definición de bienestar subjetivo hay dos aspectos centrales que deben tenerse en cuenta: el de tipo afectivo y el de tipo cognitivo (Diener, Suh, Lucas y Smith, 1999). El primero se refiere al componente emocional, por lo que los niveles de afecto positivo y negativo son los que se utilizan para indicar el nivel de bienestar. El aspecto cognitivo alude a la evaluación cognitiva consciente de la vida de cada individuo, en la que compara sus circunstancias vitales con un estándar autoimpuesto. Lo anterior es operacionalizado con el constructo Satisfacción de vida (Diener, Emmons, Larsen y Griffin, 1985).

El estudio del bienestar subjetivo, componente inmaterial de la calidad de vida, permite entonces conocer y analizar las percepciones y valoraciones que los niños, en este caso, hacen de sus principales dominios de vida, así como de sus aspiraciones y niveles de satisfacción (Diener, 2000; Diener, Tay y Oishi, 2013).

Respecto al bienestar subjetivo en niños, numerosos estudios han indicado que las relaciones con agentes tales como la familia, los amigos y los maestros, son determinantes para garantizarlo (Bratterud, Sandseter y Seland, 2012; Fattore,
Mason y Watson, 2009; Foley et al., 2012; Holder y Coleman, 2009; Thoilliez, 2011).

Se ha encontrado que el bienestar subjetivo de un niño en edad preescolar se vincula con la forma en que piensa de sí mismo, de los demás y en cómo se siente con otras personas y consigo mismo (Mihaela, 2015), lo cual, a su vez, se asocia con su capacidad de adaptación a las tareas diarias (Denham, Wyatt, Bassett, Echeverria y Knox, 2009).

El bienestar subjetivo de los niños en edad preescolar se refleja además en sus pensamientos, sentimientos y comportamientos; además incluye un cierto número de competencias necesarias para que crezcan y se adapten a la escuela y al entorno social por el resto de su vida, como la inteligencia social y la flexibilidad comportamental (Abed, Pakdaman, Heidari y Tahmassian, 2016). Dichas competencias incluyen la capacidad para expresar sentimientos e identificar o entender los sentimientos del otro, empatizar con otras personas y regular su comportamiento para ajustarse a las conductas que identifica en los demás. Hay otros factores importantes, como las emociones positivas que los niños pueden sentir (felicidad, optimismo, amor, confianza en sí mismo, curiosidad, inspiración, diversión, paz interior y otros más), que, de acuerdo con Darling-Churchill y Lippman (2016), desempeñan un importante papel en el desarrollo psicoafectivo del niño y en su bienestar emocional y social.

El desarrollo de instrumentos de medición válidos y confiables es un elemento que se ha considerado imprescindible en el bienestar subjetivo. A este respecto, se han diseñado instrumentos específicos para medirlo en la referida población, algunos de los cuales se comentan a continuación.

La encuesta Tellus, que se desarrolló para aplicarse en línea, sondeó mediante cinco preguntas las opiniones de niños ingleses de entre 7 y 10 años sobre la felicidad y las relaciones con amigos y familiares (Chamberlain, George, Golden, Walker y Benton, 2010). Por otro lado, en la misma Inglaterra, desde 1994 se ha aplicado el Cuestionario para Jóvenes del Panel de Hogares (cf. McAuley y Rose, 2010), que indaga tres dimensiones en niños de 11 a 15 años: felicidad (familia, amigos, satisfacción general, escuela), preocupación (tristeza, 
desesperanza, depresión) y autoestima (agradar a los demás y sentirse útil). Sobre la misma línea de trabajo, la Escala de Bienestar Stirling para Niños (SCWBS) se elaboró para medir el bienestar emocional y psicológico en diferentes contextos comunitarios en niños de 8 a 15 años, instrumento cuyos indicadores son el optimismo, la alegría, la capacidad, las relaciones interpersonales satisfactorias, la claridad del pensamiento y las competencias (Godfrey, Devine-Wright y Taylor, 2015).

Si bien los instrumentos mencionados han propiciado una forma objetiva de medir el bienestar psicológico (particularmente en niños mayores de 6 años y en adolescentes europeos), es evidente que la medición del bienestar en niños aún es un campo incipiente y poco explorado en niños menores de esa edad. Adicionalmente, en idioma español no existen instrumentos psicométricos válidos y confiables para evaluar el bienestar en la población infantil en el contexto latinoamericano, lo que constituye un problema en cuanto que la medición objetiva de dicha dimensión es útil para formular políticas públicas para el fomento de ciertos factores protectores de la salud mental y la prevención de factores de riesgo en situaciones negativas, particularmente con los niños que habitan en esta región del mundo (Alfaro, Casas y López, 2015). En razón de lo anterior, se considera que la versión en español de la Children Psychological Well-being Scale, o CPWS, publicada originalmente en Irán por Abed et al. (2016), puede ser una herramienta psicométrica válida y confiable para medir el bienestar subjetivo en niños latinoamericanos -específicamente colombianos-, por lo que se llevó a cabo el presente estudio para describir las propiedades psicométricas de dicha escala, probándose las siguientes hipótesis:

H1: Debido a las diferencias culturales entre Irán y Colombia, es posible que la CPWS no posea la misma estructura factorial que la obtenida en el estudio de Abed et al. (2016), por lo que se supone que la CPWS muestra una estructura unifactorial tal que sus doce ítems configuran un constructo general de bienestar psicológico infantil.

H2: Debido a que en su validación la escala CPWS presentó una estructura de tres factores latentes (Abed et al., 2016), la escala muestra la misma estructura factorial en su aplicación en
Colombia, con tres factores que pueden ser denominados autoconcepto, satisfacción con la vida y resiliencia.

\section{MÉTODO}

\section{Diseño}

Para analizar las propiedades psicométricas de la CPWS se empleó un diseño de validación mediante un muestreo por conveniencia de 229 niños $(n=229)$. La recolección de datos fue hecha de manera transversal en diez centros educativos del departamento de Córdoba (Colombia).

\section{Participantes}

En la Tabla 1 se presentan las características sociodemográficas de la muestra de infantes que participaron del estudio, $48.03 \%$ de los cuales fueron niños y $51.97 \%$ niñas, con edades de 3 a 6 años. En cuanto a los cuidadores, la mayoría fueron mujeres $(87.2 \%)$, y en unión libre con sus parejas $(51.5 \%)$.

Tabla 1. Información sociodemográfica de la muestra.

\begin{tabular}{|c|c|c|c|}
\hline Variable & Valor & n & $\%$ \\
\hline \multirow{2}{*}{ Sexo del niño(a) } & Hombre & 119 & 52.0 \\
\hline & Mujer & 110 & 48.0 \\
\hline \multirow{4}{*}{ Edad del niño(a) } & 3 & 38 & 16.59 \\
\hline & 4 & 72 & 31.44 \\
\hline & 5 & 79 & 34.50 \\
\hline & 6 & 40 & 17.47 \\
\hline \multirow{2}{*}{ Sexo del cuidador } & Hombre & 28 & 12.2 \\
\hline & Mujer & 201 & 87.2 \\
\hline \multirow{5}{*}{ Estado civil del cuidador } & Soltero & 23 & 10.1 \\
\hline & Casado & 76 & 33.5 \\
\hline & Separado & 8 & 3.5 \\
\hline & Unión libre & 117 & 51.5 \\
\hline & Viudo & 3 & 1.3 \\
\hline \multirow{5}{*}{$\begin{array}{l}\text { Nivel educativo } \\
\text { del cuidador }\end{array}$} & Primaria & 23 & 10.1 \\
\hline & Secundaria & 73 & 32.2 \\
\hline & Tecnología & 63 & 27.8 \\
\hline & Profesional & 36 & 15.9 \\
\hline & Posgrado & 32 & 14.1 \\
\hline
\end{tabular}




\section{Instrumentos}

La escala Children Well-Being Scale (CPWS) (Abed et al., 2016) consta de doce ítems que se evalúan mediante una escala Likert de tres anclajes. Los puntajes de cada ítem corresponden a "Sí" (dos puntos), "Algunas veces" (un punto), y "No" (sin puntos). La escala fue traducida y retrotraducida del inglés al español. Un ejemplo de ítem es "Soy feliz cuando estoy con mi familia" (ver Anexo).

Además, se dispuso de una ficha sociodemográfica que incluyó las variables de edad, sexo y condición de afiliación al sistema de salud.

\section{Procedimiento}

Teniendo en cuenta las consideraciones éticas respectivas para la investigación en niños pequeños en Colombia (cf. Congreso de Colombia, 2006; Ministerio de Salud, 1993), se estableció contacto con los centros educativos para acceder a la muestra y obtener el correspondiente consentimiento informado por parte de los representantes legales de los niños. Al final de cada sesión de evaluación los cuestionarios fueron revisados junto con los participantes para asegurar de que hubieran sido completados en su totalidad.

\section{Análisis estadísticos}

El análisis de datos fue llevado a cabo empleando el IBM SPSS, v. 24, y RStudio, mismos que consistieron en la obtención de estadísticos descriptivos, análisis de dimensionalidad de los modelos a tra- vés de análisis factorial confirmatorio, análisis de correlaciones de Pearson, así como diversos análisis de varianza (ANOVA) y de pruebas $t$ de los factores de la CPWS, según la información sociodemográfica proporcionada por los participantes.

\section{RESULTADOS}

\section{Estadísticos descriptivos}

El puntaje promedio obtenido por los participantes en la CPWS fue relativamente alto $(M=21.79$; $\sigma=2.62$ ). Tras efectuar una prueba Kolmogorov-Smirnov, fue posible identificar que los puntajes de la escala no siguieron una distribución normal.

\section{Análisis de dimensionalidad}

Se analizó la estructura factorial de la escala CPWS mediante el contraste del ajuste de dos modelos teóricos, utilizando para ello análisis factoriales confirmatorios.

En primer lugar, el modelo 1 asocia los doce ítems de la CPWS a un único factor subyacente, de manera que teóricamente la CPWS evalúa un constructo de bienestar psicológico general $(\mathrm{M}=21.79$, $\sigma=2.62$ ) (Figura 1). Los coeficientes path del modelo indican que las variables se relacionan con el factor bienestar psicológico general con una magnitud de mínimo $\beta=.14$ (CPWS 9) y de máximo $\beta=.78$ (CPWS 12).

Figura 1. Diagrama path del modelo 1 (unifactorial).

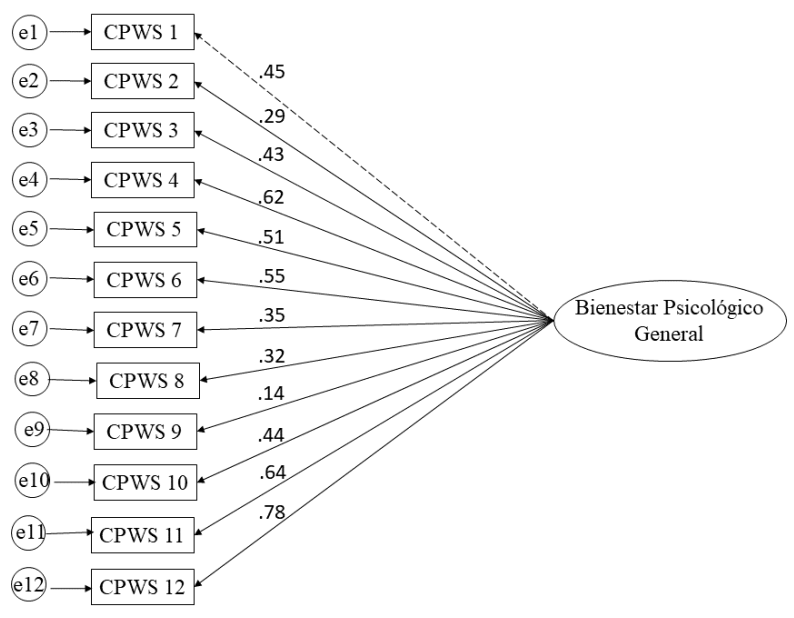


El modelo 2 asocia los doce ítems de la CPWS según el modelo teórico hipotetizado por Abed et al. (2016) (Tabla 2), en el que la escala tiene una es- tructura de tres factores: autoconcepto $(\mathrm{M}=4.87$, $\sigma=1.42)$, satisfacción con la vida $(\mathrm{M}=6.65$, $\sigma=1.22)$ y resiliencia $(\mathrm{M}=2.69, \sigma=2.62)$ (Figura 2).

Tabla 2. Factores que componen la CPWS según el modelo teórico de Abed et al. (2016) y los ítems que los componen.

\begin{tabular}{|c|c|c|}
\hline Factor & Índices & Ítem \\
\hline \multirow{4}{*}{ Autoconcepto } & CPWS 1 & Me gusto a mí mismo. \\
\hline & CPWS 2 & Soy simpático(a). \\
\hline & CPWS 3 & Me gustan mis amigos. \\
\hline & CPWS 4 & Soy feliz cuando estoy con mis amigos. \\
\hline \multirow{6}{*}{$\begin{array}{l}\text { Satisfacción } \\
\text { con la vida }\end{array}$} & CPWS 5 & Me gusta mi familia. \\
\hline & CPWS 6 & Soy feliz cuando estoy con mi familia. \\
\hline & CPWS 7 & Soy una persona feliz. \\
\hline & CPWS 10 & Soy importante para mis padres. \\
\hline & CPWS 11 & Tengo una buena vida y estoy feliz con ella. \\
\hline & CPWS 12 & Cuál de estas caras tienes en la mayor parte del tiempo. \\
\hline \multirow[t]{2}{*}{ Resiliencia } & CPWS 8 & $\begin{array}{l}\text { Cuando me ocurre un problema (por ejemplo, se me rompe } \\
\text { un juguete favorito), me calmo pronto. }\end{array}$ \\
\hline & CPWS 9 & Cuando me ocurre un problema puedo resolverlo. \\
\hline
\end{tabular}

Fuente: Abed et al. (2016).

Figura 2. Diagrama path del modelo 2 (tres factores).

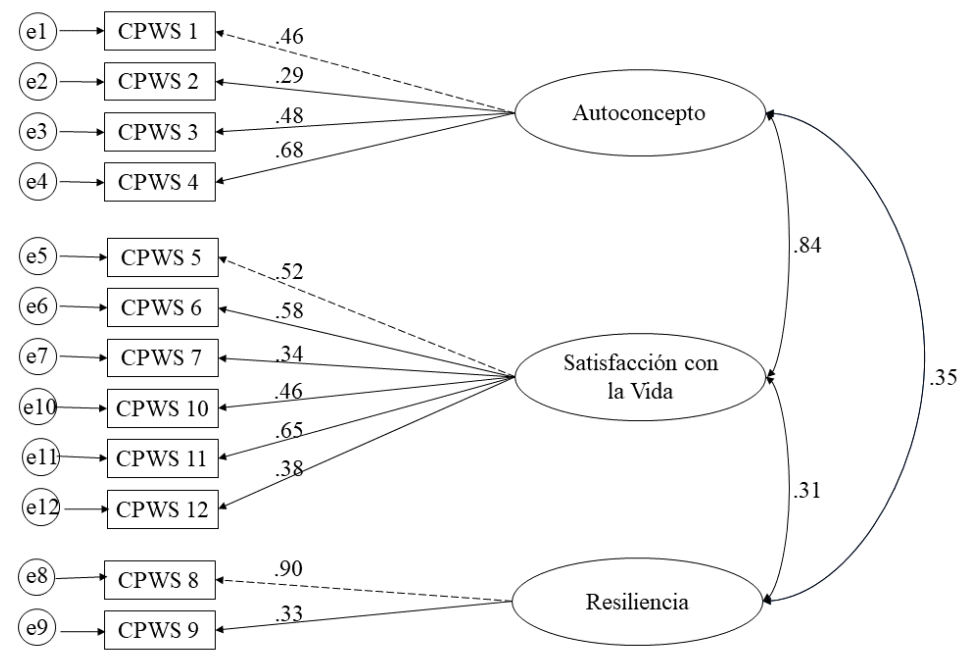

Los coeficientes path del modelo 2 indican que las variables se relacionan con sus respectivos factores con una magnitud de mínimo $\beta=.29$ en el caso del ítem CPWS 2 con el factor Autoconcepto; y de máximo $\beta=.90$ en el caso del ítem CPWS 8 con el factor Resiliencia. El diagrama path también señala la correlación entre los factores, de manera que Autoconcepto tiene una correlación positiva de .35 con el factor Resiliencia, y de .84 con el factor Satisfacción con la Vida. Adicionalmente, el factor Satisfacción con la Vida muestra una correlación de .31 con el factor Resiliencia.

Para evaluar los índices de ajuste de los dos modelos teóricos, se calcularon los indicadores $\chi^{2}$, SRMR, CFI y RMSEA para cada modelo (Tabla 3 ), y para interpretar los resultados se empleó el criterio de Hu y Bentler (1999), quienes plantean una combinación de SRMR (buen ajuste $<.09$ ) y RMSEA (buen ajuste de .06), o bien una combinación de CFI (buen ajuste $\approx .95$ ) y SRMR (buen ajuste $<.09$ ). 
Tabla 3. Índices de ajuste de los dos modelos evaluados mediante análisis factorial confirmatorio.

\begin{tabular}{|c|c|c|c|c|c|c|c|}
\hline Modelo & $\mathbf{N}$ & $\boldsymbol{X}^{\mathbf{2}}$ & g.l. & $\boldsymbol{p}$ & SRMR & CFI & RMSEA \\
\hline Modelo 1 & 229 & 139.691 & 54 & $<.001$ & .068 & .775 & .083 \\
\hline Modelo 2 & 229 & 116.548 & 51 & $<.001$ & .061 & .090 & .050 \\
\hline
\end{tabular}

Los resultados indican que el modelo 1 no presenta un buen ajuste (ver Tabla 3), a diferencia del modelo 2, que sí lo hace, teniendo en cuenta los valores del SRMR y del RMSEA (Escobedo, Hernández, Estebané y Martínez, 2016; Hu y Bentler, 1999). Por lo tanto, es recomendable apegarse a la estructura teórica propuesta por Abed et al. (2016) en el análisis de varianza según la información sociodemográfica.

\section{Análisis de correlaciones}

Se elaboró una matriz de correlaciones de Pearson para analizar las asociaciones entre los factores que componen la escala CPWS y las variables sociodemográficas ordinales de la muestra (Tabla 4).

Tabla 4. Correlaciones entre los factores del CPWS y las variables sociodemográficas ordinales.

\begin{tabular}{|l|c|c|c|}
\hline \multicolumn{1}{|c|}{ Factores } & Autoconcepto & Satisfacción & Resiliencia \\
\hline Estrato & $-.257^{* *}$ & $-.199^{* *}$ & -.072 \\
\hline Edad del infante & $.133^{*}$ & -.012 & .057 \\
\hline Edad del cuidador & -.001 & -.034 & -.110 \\
\hline
\end{tabular}

Las correlaciones sugieren que el estrato socioeconómico presenta una asociación significativamente negativa con los factores Autoconcepto $(\mathrm{r}=-.257, p<.01)$ y Satisfacción $(\mathrm{r}=-.199)$, así como con el puntaje general de la escala CPWS $(\mathrm{r}=-.26, p<.01)$. En contraste, Estrato socioeconómico no mostró una correlación significativa con Resiliencia.

Por otra parte, la edad del infante mostró una asociación significativa y positiva con Autoconcepto $(\mathrm{r}=.133, p<.05)$; en cambio, esta variable no se asoció significativamente con los factores Satisfacción con la Vida y Resiliencia. Finalmente, la edad del cuidador no mostró ninguna asociación estadísticamente significativa con los factores de la CPWS.

\section{Diferencia de medias}

Una serie de pruebas $t$ y ANOVA permitió analizar si los puntajes en cada uno de los factores de la CPWS difieren según las variables sociodemográficas categóricas: estatus marital, personas con quienes vive, nivel de estudio de los cuidadores, ocupación laboral, género de los padres y género de los infantes. Es interesante constatar que los infantes a cargo de hombres manifestaron niveles significativamente más altos de resiliencia $(\mathrm{M}=3.11)$, a diferencia de los infantes a cargo de mujeres $(\mathrm{M}=2.64), t(37)=2.58, p=.013$. Este contraste tiene un tamaño del efecto mediano, con una $g$ de Hedges de 0.48 . No se halló ninguna otra diferencia según las variables sociodemográficas en los factores de la CPWS.

\section{DISCUSIÓN}

Éste es el primer estudio que ha utilizado las propiedades psicométricas de la Children Well-Being Scale (CPWS) para evaluar niños colombianos de 3 a 6 años, utilizando para ello un diseño de validación. La escala muestra propiedades psicométricas aceptables sobre la validez de constructo y contenido. Sin embargo, en el cálculo de la consistencia interna por cada una de las dimensiones se obtuvieron indicadores menores a los reportados por Abed et al. (2016), aspecto que podría estar relacionado con factores culturales; tal como es el caso de los niños colombianos de este estudio y los niños iraníes de la ciudad de Teherán, de la investigación de Abed et al. A este respecto, sería interesante replicar el presente estudio con muestras poblacionales de otras regiones colombianas.

Cuatro conclusiones generales pueden desprenderse de este trabajo. 
En primer lugar, los análisis factoriales confirmatorios realizados apoyan la estructura factorial de la CPWS propuesta en la validación original de Abed et al. (2016). Adicionalmente, los indicadores psicométricos obtenidos en la traducción colombiana se corresponden con los criterios de bienestar psicológico en niños definidos por la teoría que sustenta este tipo de instrumentos (Chamberlain et al., 2010; Godfrey et al, 2015; Ravens-Sieberer, Karow, Berthel y Klasen, 2014).

En segundo término, el estrato socioeconómico de los niños se asoció negativamente con Autoconcepto y Satisfacción con la Vida, pero no con Resiliencia. Este resultado es llamativo en cuanto que sugiere que los niños que viven en localidades con un estrato social superior manifiestan niveles de autoconcepto y satisfacción con la vida significativamente menores que los de estratos sociales inferiores. En contraste, Resiliencia no se asoció significativamente con Estrato social.

En tercer lugar, la edad de los niños se asoció positivamente con el autoconcepto, pero no con Satisfacción con la Vida ni con Autoestima. Este resultado indica que la CPWS permite discriminar entre constructos que dependen del desarrollo psicológico del niño, y establecen un precedente sobre la posibilidad de que en futuros estudios surjan amenazas a la validez por efecto de maduración del participante. Notablemente, la edad del cuidador no tuvo ninguna asociación estadísticamente significativa con los factores de la CPWS.

En cuarto término, el género de los infantes no se asoció con los niveles de ninguno de los factores de la CPWS, lo que sugiere que no hubo diferencias de género de los infantes respecto a su bienestar psicológico. No obstante, los niños acompañados por cuidadores varones exhibieron niveles de resiliencia significativamente mayores que aquellos acompañados por cuidadores mujeres, resultado que muestra que las variables sociodemográficas de los cuidadores están vinculadas de manera particular a la resiliencia de los infantes, pero no al autoconcepto o a la satisfacción con la vida.

Por último, otras características de los cuidadores, como el estatus marital, las personas con las que vive, su nivel de estudio y ocupación laboral no fueron variables vinculadas de manera estadísticamente significativa con los factores de la CPWS.
El presente estudio tiene algunas limitaciones que deben subrayarse. En primer lugar, hay una diferencia metodológica frente al estudio de validación de Abed et al. (2016), ya que la encuesta fue autoaplicada y supervisada por el docente a cargo del niño, pero no se reportó si los participantes eran competentes en habilidades de lectoescritura. En segundo lugar, aquí se utilizó un muestreo por conveniencia según la disponibilidad de acceso a los cuidadores de los niños en las diez instituciones educativas, por lo que se dificulta la posibilidad de generalizar los hallazgos al resto de la población. En tercer lugar, la mayoría de los cuidadores de los participantes fueron mujeres, una variable asociada a los puntajes de resiliencia. El hecho de tener una sobrerrepresentación de cuidadores mujeres puede introducir un sesgo en la asociación entre la resiliencia y el género del cuidador.

Para un futuro curso de la investigación se recomienda desarrollar análisis complementarios con otras muestras poblacionales colombianas de diferentes estratos socioeconómicos, e igualmente efectuar un análisis test-retest de la CPWS para probar la fiabilidad de la escala. También es recomendable incluir la misma proporción de cuidadores hombres y mujeres para verificar si esta variable se relaciona con la resiliencia de los infantes. Por último se sugiere aplicar la CPWS junto con otras escalas que, según la literatura existente, están relacionadas con el fin de realizar una validación convergente de la escala que permita ampliar la inferencia sobre su validez de constructo.

Para finalizar, es posible concluir que la versión colombiana de la Children Well-Being Scale es un instrumento que puede ser útil para identificar niveles de bienestar en niños, por lo que se sugiere su uso en futuros estudios descriptivos sobre el bienestar subjetivo en niños colombianos en general, en comparaciones entre estratos, niveles educativos y demás variaciones que hagan posible dar cuenta de su utilidad en la citada población. No obstante, para que esta escala pueda convertirse en una herramienta de uso práctico en los contextos clínico, educativo e investigativo, debe soportar un proceso de validación que compense las limitaciones señaladas y que reporte indicadores psicométricos más cercanos a los obtenidos por Abed et al. (2016). 


\section{REFERENCIAS}

Abed, N., Pakdaman, S., Heidari, M. y Tahmassian, K. (2016). Developing psychological well-being scale for preschool children. Global Journal of Health Science, 8(11), 104-111.

Alfaro, J., Casas, F. y López, V. (2015). Bienestar en la infancia y la adolescencia. Psicoperspectivas: Individuo y Sociedad, 14(1), 1-5.

Bratterud, A., Sandseter, E. y Seland, M. (2012). Children's wellbeing and participation in kindergarten. The perspectives of children, parents and staff. Trondheim: NTNU Samfunnsforskning.

Chamberlain, T., George, N., Golden, S., Walker, F. y Benton, T. (2010). Tellus4 National Report. London: National Foundation for Educational Research. Recuperado de http://collectionsr.europarchive.org/tna/20111108225525.

Congreso de Colombia (2006). Ley 1090 “por la cual se reglamenta el ejercicio de la profesión de Psicología, se dicta el Código Deontológico y Bioético y otras disposiciones”. Bogotá (Colombia): Autor. Recuperado de http://www.funcionpublica. gov.co/eva/gestornormativo/norma_pdf.php? $\mathrm{i}=66205$.

Darling-Churchill, K. y Lippman, L. (2016). Early childhood social and emotional development: Advancing the field of measurement. Journal of Applied Developmental Psychology, 45, 1-7.

Denham, S., Wyatt, T., Bassett, H., Echeverria, D. y Knox, S. (2009). Assessing socio- emotional development in children from a longitudinal perspective. Journal of Epidemiology and Community Health, 63, 37-52.

Diener, E. (1984). Subjective well-being. Psychological Bulletin, 95, 542-575.

Diener, E. (2000). Subjective well- being: The science of happiness and a proposal for a national index. American Psychologist, 55(1), 34-43. doi: 10.1037/0003-066X.55.1.3.

Diener, E., Emmons, R., Larsen, R. y Griffin, S. (1985). The Satisfaction with Life Scale. Journal of Personality Assessment, 49(1), 71-75.

Diener, E., Suh, E., Lucas, R. y Smith, H. (1999). Subjective well-being: Three decades of progress. Psychological Bulletin, 125, 276-302.

Diener, E., Tay, L. y Oishi, S., (2013). Rising income and subjective well-being of nations. Journal of Personality and Social Psychology, 104(2), 267-276. doi: 10.1037/a003048.

Escobedo, M., Hernández, J., Estebané, V. y Martínez, G. (2016). Modelos de ecuaciones estructurales: Características, fases, construcción, aplicación y resultados. Ciencia y Trabajo, 18(55), 16-22. doi: http://dx.doi. org/10.4067/S071824492016000100004.

Fattore, T., Mason, J. y Watson, E. (2009). When children are asked about their well-being: Towards a framework for guiding policy. Child Indicators Research, 2(1), 57-77. doi: 10.1007/s12187-008-9025-3.

Foley, K., Blackmore, A., Gidler, S., Donnell, M., Glauert, R. y Llewellyn, G. (2012). To feel belonged: the voices of children and youth with disabilities on the meaning of wellbeing. Child Indian Research, 5, 375-391. doi: 10.1007/s12187-011-9134-2.

Godfrey, C., Devine-Wright, H. y Taylor, J. (2015). The positive impact of structured surfing courses on the well-being of vulnerable young people. Community Practitioner, 88(1), 26-29.

Holder, M.D. y Coleman, B. (2009). The contribution of social relationships to children's happiness. Journal of Happiness Studies, 10, 329-349.

Hu, L. y Bentler, P.M. (1999). Cutoff criteria for fit indexes in covariance structure analysis: Conventional criteria versus new alternatives. Structural Equation Modeling: A Multidisciplinary Journal, 6(1), 1-55.

McAuley, C. y Rose, W. (2010). Child well-being: understanding children'lives. London: Jessica Kingsley Publishers.

Mihaela, I. (2015). Promoting the emotional wellbeing of preschoolers. Procedia: Social and Behavioral Sciences, 209, $509-513$.

Ministerio de Salud (1993). Resolución 8430, por la cual se establecen las normas científicas, técnicas y administrativas para la investigación en salud. Bogotá (Colombia): Autor. Recuperado de https://www.minsalud.gov.co/sites/rid/Lists/BibliotecaDigital/RIDE/DE/DIJ/RESOLUCION-8430-DE-1993.PDF.

Ravens-Sieberer, U., Karow, A., Berthel, D. y Klasen, F. (2014). How to assess quality of life in child and adolescent psychiatry. Dialogues in Clinical Neuroscience, 16(2), 147-158.

Thoilliez, B. (2011). How to grow up happy: an exploratory study on the meaning of happiness from children's voices. Child Indicators Research, 4(2), 323-351. 
Anexo

Escala de Bienestar Psicológico para Niños (CPWS)

Nombre:

Edad:

\begin{tabular}{|c|c|c|c|}
\hline 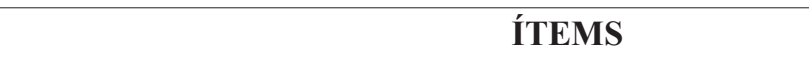 & Factor 1 & Factor 2 & Factor 3 \\
\hline \multicolumn{4}{|l|}{ 1. Me gusto a mí mismo(a). } \\
\hline \multicolumn{4}{|l|}{ 2. Soy simpático(a). } \\
\hline \multicolumn{4}{|l|}{ 3. Me gustan mis amigos. } \\
\hline \multicolumn{4}{|l|}{ 4. Soy feliz cuando estoy con mis amigos. } \\
\hline \multicolumn{4}{|l|}{ 5. Me gusta mi familia. } \\
\hline \multicolumn{4}{|l|}{ 6. Soy feliz cuando estoy con mi familia. } \\
\hline \multicolumn{4}{|c|}{ 7. Soy una persona feliz. } \\
\hline \multicolumn{4}{|c|}{$\begin{array}{l}\text { 8. Cuando me ocurre un problema (por ejemplo, se me rompe un juguete favorito) } \\
\text { me calmo pronto. }\end{array}$} \\
\hline \multicolumn{4}{|c|}{ 9. Cuando me ocurre un problema, puedo resolverlo. } \\
\hline \multicolumn{4}{|l|}{ 10. Soy importante para mis padres. } \\
\hline \multicolumn{4}{|l|}{ 11. Tengo una buena vida y estoy feliz con mi vida. } \\
\hline 12. ¿Cuál de estas caras tienes la mayor parte del tiempo? & & & \\
\hline
\end{tabular}

